\title{
Analysis of the Performance of a Dehulling System for Confectionary Sunflower Seeds
}

\author{
Ana Karina de Figueiredo, Luciana Marcela Rodríguez, Isabel Concepción Riccobene, \\ Susana Maria Nolasco* \\ Facultad de Ingeniería, Universidad Nacional del Centro de la Provincia de Buenos Aires, Grupo de \\ Investigación TECSE, Departamento de Ingeniería Química, Buenos Aires, Argentina \\ Email: snolasco@fio.unicen.edu.ar
}

Received 11 November 2013; revised 11 December 2013; accepted 18 December 2013

Copyright (C) 2014 by authors and Scientific Research Publishing Inc.

This work is licensed under the Creative Commons Attribution International License (CC BY). http://creativecommons.org/licenses/by/4.0/

(c) (i) Open Access

\begin{abstract}
Confectionary sunflower, which has a larger size and lower oil content than oilseed sunflower, has a considerable market, since it is used for birdfeed and human consumption. The characteristics of use of confectionary sunflower seeds require a dehulling process that is efficient in the removal of the hull and that also allows obtaining a product consisting mainly of whole kernels. Response surface methodology was used to determine an optimal combination of working conditions in the dehulling process of confectionary sunflower seeds. Optimization factors were impact speed, expressed in terms of the peripheral speed $(28.3-41.9 \mathrm{~m} / \mathrm{s})$, and moisture content of the seeds $(4 \%$ $14 \%$, dry basis-db). A central composite rotatable design (CCRD) was used to develop models for the responses (dehulling ability-DA and percentage of whole kernels-WK). Applying the desirability function method, the optimal values of the factors were determined using maximum $W K$ and maximum $D A$ as criteria. The results of the optimization technique suggest that by dehulling Mycogen 9338 confectionary sunflower seeds at $12.3 \% \mathrm{db}$ and $32.5 \mathrm{~m} / \mathrm{s}$, the maximum values of $D A$ and $W K(72.6 \%$ and $63 \%$, respectively) would be obtained. The moisture value defined as optimal determines a requirement of humidification of the seeds prior to dehulling, establishing the need for a technical and economic feasibility study.
\end{abstract}

\section{Keywords}

Confectionary Sunflower; Dehulling; Response Surface Methodology; Whole Kernels

\section{Introduction}

Sunflower is mainly cultivated for commercial oilseed production by pressing and/or solvent extraction. Al*Corresponding author. 
though the non-oilseed variety (confectionary sunflower, of larger size and lower oil content) is grown to a lesser extent, it has a wide market because it is used for human consumption and in the food industry for birds and others animals. Confectionary sunflower is generally classified into three categories: the larger seeds are roasted, salted and packaged for human consumption; medium size seeds are dehulled and packaged for use as snacks or in bakery foods; and the smaller seeds are used as poultry feed.

Commercial processing of sunflower seeds requires achieving an efficient separation of the hull from the seed in the dehulling process. In the oil industry, partial dehulling of the seed presents many advantages, such as a suitable bulk porosity for the oil extraction process, better quality of both raw oil (lower wax content) and de-oiled meal (higher protein content), as well as an increase of the life span of the machinery [1] [2]. On the other hand, the size of confectionary sunflower seeds and the easiness to remove their hull are two properties that have an essential role in the quality of the obtained product. The characteristics of use of this seed require a dehulling process that is efficient in the removal of the hull and that also allows obtaining a product consisting mainly of whole kernels.

The most efficient method for the industrial processing of sunflower seeds is based on a combination of impact and centrifugal forces. During impact dehulling, seeds are fed into the top of the equipment and a spinning rotor expels them against the impact frame of the dehuller. The force of the impact causes the hull to break away from the kernel, and then the hull is removed by aspiration as the kernels are separated by vibratory sieves.

Many investigations have shown that the dehulling ability depends on different characteristics of the seeds, such as size and density [1]-[3], hull content [4] [5], oil content [3] [6] and moisture content [2] [3] [7]-[9]. Subramanian et al. [2] and Gupta and Das [10] analyzed the effect of feed rate and impeller speed on the dehulling performance of oilseed sunflower varieties grown in India. Previous studies investigated the effect of moisture content of the seeds on the dehulling ability of a non-oilseed sunflower hybrid [8], but a search of the literature does not show any studies on confectionary sunflower involving other process variables or a combined study of those variables. Therefore, the aim of the present work was to determine an optimal combination of the operating conditions (moisture content of the seeds and dehuller impact speed) in the dehulling process in order to maximize the dehulling ability maintaining a high percentage of whole kernels.

\section{Materials and Methods}

\subsection{Sample Preparation}

The confectionary variety Mycogen 9338 (Morgan) was selected for this study. The sunflower seeds used were grown in Olavarría $\left(36.5^{\circ} \mathrm{S}, 60.5^{\circ} \mathrm{W}\right)$, Buenos Aires province, Argentina. The seeds were manually cleaned to remove all foreign matter, broken or immature seeds. The moisture content of the seeds (MC) was determined according to the ASAE S352.2 Method [11]. Both the hull total content (H total) and the kernel total content (WKtotal) were determined by manual dehulling from a sample of $10 \mathrm{~g}$ and were expressed as dry basis percentage (\%, d.b.). The desired moisture content was obtained by drying the seeds in a convection air oven at $40^{\circ} \mathrm{C}-45^{\circ} \mathrm{C}$ or by spraying with pre-calculated amounts of distilled water, and then thoroughly mixing and sealing the samples in separate polyethylene bags. The samples were kept in a refrigerator for at least $72 \mathrm{~h}$ to allow a homogeneous moisture distribution. Before starting a test, the required amount of seeds was taken out of the refrigerator and allowed to equilibrate to ambient temperature.

\subsection{Dehulling Ability and Whole Kernel Percentage}

The mechanical dehulling was carried out by impact in a dehulling pilot equipment based on a centrifugal process (Figure 1). The rotor speed was adjusted with a variable frequency drive and calibrated with a tachometer. After the mechanical dehulling of the sample, the resulting product was classified into hull, whole kernels and rest (undehulled seeds, partially dehulled seeds, broken kernels and fines, i.e. material smaller than $2 \mathrm{~mm}$ ). The fractions were dried in a forced-air oven at $130^{\circ} \mathrm{C}$ for $3 \mathrm{~h}$, and the mechanically-extracted hull/kernel percentages were calculated by Equations (1) and (2), respectively, expressed as percentages in dry basis.

$$
H_{\text {mec }}=\frac{\text { total hull extracted }(\mathrm{g})}{\text { total hull extracted }(\mathrm{g})+\text { Whole kernel extracted }(\mathrm{g})+\text { rest }(\mathrm{g})}
$$




$$
W K_{\text {mec }}=\frac{\text { Whole kernel extracted }(\mathrm{g})}{\text { total hull extracted }(\mathrm{g})+\text { Whole kernel extracted }(\mathrm{g})+\text { rest }(\mathrm{g})} \times 100
$$

The dehulling ability $(D A)$ and the whole kernels production $(W K)$ were determined as the percentage ratio of the percentage of mechanically extracted hull/whole kernels to the total amount of hull/kernels of the seeds, Equations (3) and (4), respectively.

$$
\begin{gathered}
D A(\%)=\frac{H_{m e c}}{H_{\text {total }}} \times 100 \\
W K(\%)=\frac{W K_{\text {mec }}}{W K_{\text {total }}} \times 100
\end{gathered}
$$

\subsection{Experimental Design and Statistical Analysis}

The response surface methodology (RSM) combined with a central composite rotatable design (CCRD) were used to point out the relationship between the response functions and the process variables, as well as to determine the conditions of these variables able to optimize the performance of the dehulling system for confectionary sunflower seeds. The design consisted of 11 experiments with three center points and four axial points added to the $2^{2}$ full factorial design (Table 1). All the experiments were conducted in triplicate. The impact dehuller rotor speed (rpm) was expressed in terms of the peripheral speed (PS) of the impeller $(\mathrm{m} / \mathrm{s})$.

STATGRAPHICS Centurion XV software (Version 15.2.06, StatPoint, Inc.) was used to perform the statistical analysis of the results, develop multiple regression models from experimental data, and predict process conditions that improve the performance of the dehulling system. Experimental data were fitted to a generalized second-order model by the following equation:

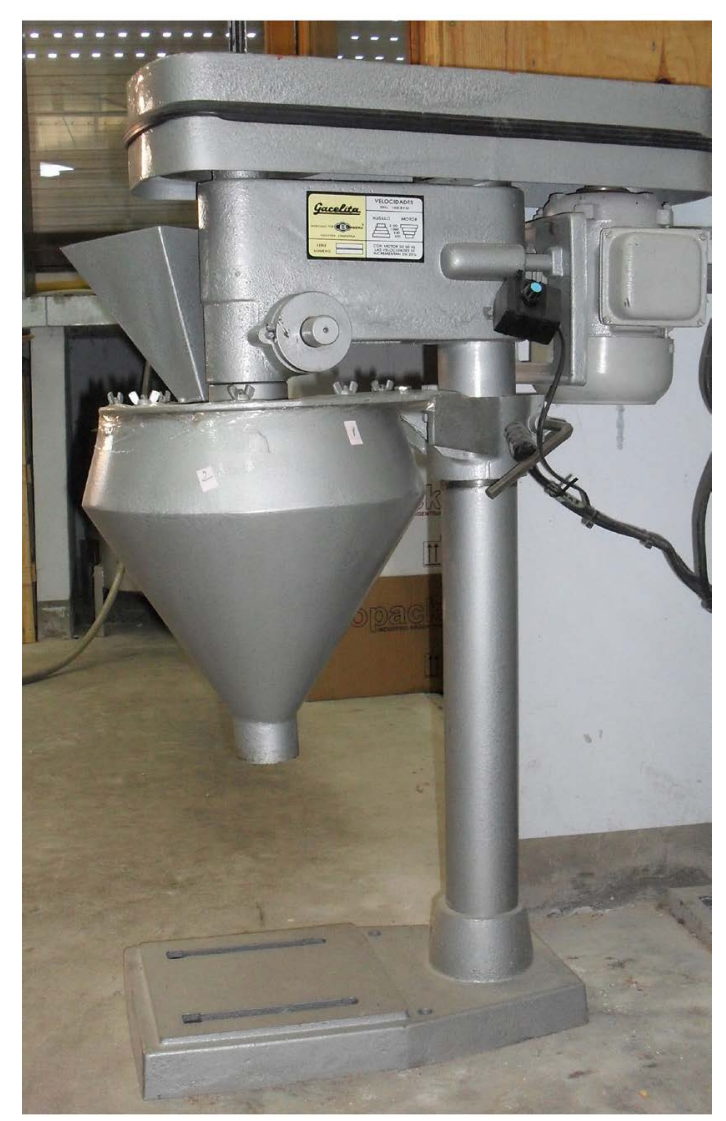

Figure 1. Dehulling pilot equipment. 
Table 1. Experimental program and experimental mean values of response variables for central composite rotatable design.

\begin{tabular}{ccccccc}
\hline \multirow{2}{*}{ Run } & \multicolumn{2}{c}{ Coded levels } & \multicolumn{2}{c}{ Actual levels } & DA & WK \\
\cline { 2 - 5 } & $X_{1}$ & $X_{2}$ & MC (\%, d.b.) & PS (m/s) & (\%, d.b.) & (\%, d.b.) \\
\hline 1 & -1 & -1 & 6 & 30.4 & $79.76(2.79)$ & 38.11(2.96) \\
2 & -1 & 1 & 6 & 38.7 & $91.91(2.66)$ & $14.77(0.84)$ \\
3 & 1 & -1 & 12 & 30.4 & $70.97(4.21)$ & $57.89(2.11)$ \\
4 & 1 & 1 & 12 & 38.7 & $79.69(4.82)$ & $38.35(1.21)$ \\
5 & -1.41 & 0 & 4.76 & 34.6 & $85.73(1.47)$ & $20.58(1.68)$ \\
6 & 1.41 & 0 & 13.2 & 34.6 & $75.24(4.20)$ & $62.55(1.35)$ \\
7 & 0 & -1.41 & 9 & 28.6 & $61.55(0.00)$ & $59.27(0.90)$ \\
9 & 0 & 1.41 & 9 & 40.5 & $85.67(2.77)$ & $14.62(0.00)$ \\
11 & 0 & 0 & 9 & 34.6 & $82.69(1.47)$ & $53.84(3.47)$ \\
\end{tabular}

$X_{1}$ (moisture content, \% d.b.); $X_{2}$ (peripheral speed, $\mathrm{m} / \mathrm{s}$ ) (Standard deviation in parentheses).

$$
Y_{i}=b_{0}+b_{1} X_{1}+b_{2} X_{2}+b_{11} X_{1}^{2}+b_{22} X_{2}^{2}+b_{12} X_{1} X_{2}
$$

where $Y_{i}$ is one of the two predicted responses (dehulling ability of the seeds or the percentage of whole kernels) and $X_{1}, X_{2}$ represent the coded levels of the independent variables.

\subsection{Optimization}

In the present work, the optimization procedure was carried out by using the desirability function (D) combined with response surface methodology. Desirability optimization methodology is based on the idea that the quality of a product or process that has multiple characteristics, when one of them is outside of some "desired" limits, is completely unacceptable. The method finds operating conditions that provide the "most desirable" response values. The optimal values of the factors are determined from the maximization of the function. A high value of $\mathrm{D}$, which varies between zero and one, indicates the best combinations of factors to optimize the system studied [12]-[14]. In the present study, D was developed for the maximum DA and maximum WK criteria.

\section{Results and Discussion}

\subsection{Fitting Models}

Results of different dehulling runs are shown in Table 1. Estimated values of regression coefficients and their corresponding p-values for the DA response are presented in Table 2. They demonstrate that moisture content of the seeds $\left(X_{1}\right)$ and peripheral speed $\left(X_{2}\right)$ had the largest effect on $D A$. These effects were followed by the quadratic term of peripheral speed $\left(X_{2}\right)$. The quadratic term of moisture content $\left(X_{1}\right)$ and the interaction between moisture content of the seeds and peripheral speed $\left(X_{1} X_{2}\right)$ did not present a significant effect on $D A(p>0.05)$.

The estimated values of regression coefficients and their corresponding p-values for the $W K$ response are shown in Table 3. All the coefficients were significant $(\mathrm{p} \leq 0.05)$, except for the moisture content of the seeds/ impact speed interaction $\left(X_{1} X_{2}\right)$.

The prediction models were rearranged by removing the terms that were not significant at $5 \%$ in the second-order polynomial models, and the corresponding equations are shown in Table 4.

ANOVA tests revealed that the quadratic polynomial models adequately represent the responses of $D A$ and WK with coefficients of determination, $\mathrm{R}^{2}=0.8282$ and $\mathrm{R}^{2}=0.9467$, respectively (Table 4). $\mathrm{R}^{2}$ and the adjusted $\mathrm{R}^{2}$ values for the studied response variables were equal or higher than 0.80 , hence there was a close agreement between the experimental results and the theoretical values predicted by the proposed models. The model adequacy was tested using the lack of fit test, which was not significant for $\mathrm{p}>0.05$. Since the models were found 
Table 2. Regresion coefficients of the development model for the dehulling ability.

\begin{tabular}{cccccccc}
\hline & $b_{0}$ & $b_{1}$ & $b_{2}$ & $b_{11}$ & $b_{22}$ & $b_{12}$ \\
\hline$b_{i}$ & 81.29 & -4.49 & 6.88 & 0.49 & -2.97 & -0.86 \\
p-value & & 0.001 & 0.000 & 0.688 & 0.026 & 0.555 \\
\hline
\end{tabular}

Table 3. Regression coefficients of the development model for the whole kernel percentage.

\begin{tabular}{ccccccc}
\hline & $b_{0}$ & $b_{1}$ & $b_{2}$ & $b_{11}$ & $b_{22}$ & $b_{12}$ \\
\hline$b_{i}$ & 49.71 & 12.86 & -13.27 & -4.58 & -6.90 & 0.95 \\
p-value & & 0.000 & 0.000 & 0.006 & 0.000 & 0.577 \\
\hline
\end{tabular}

Table 4. Adjusted models with only significant coefficients.

\begin{tabular}{ccc}
\hline Response & $D A(\%$, d.b. $)$ & $W K(\%$, d.b. $)$ \\
\hline Model equation & $D A=81.75-4.49 X_{1}+6.88 X_{2}-3.11 X_{2}^{2}$ & $W K=49.71+12.86 X_{1}-13.27 X_{2}-4.58 X_{1}^{2}-6.90 X_{2}^{2}$ \\
Model (p-value) & $<0.0001$ & $<0.0001$ \\
$\mathrm{R}^{2}$ & 0.8282 & 0.9467 \\
Adjusted $\mathrm{R}^{2}$ & 0.7996 & 0.9341 \\
Lack of fit (p-value) $^{\mathrm{a}}$ & 0.6308 & 0.7643 \\
\hline
\end{tabular}

${ }^{\mathrm{a}}$ Want the selected model to have non-significant lack of fit $(\mathrm{p}>0.05)$.

to show insignificant lack of fit (Table 4), the responses were sufficiently explained by the regression equations.

The coefficients in Table 4 are presented in terms of coded factors. Thus, the size of the coefficients could directly be related to the observed change in the response, enabling a straight comparison between the coefficients. Accordingly, the importance of each factor in the response could be evaluated. The coefficients of moisture content $\left(X_{1}\right)$ were, in absolute value, in the same order as those of peripheral speed $\left(X_{2}\right)$, indicating that both factors had the same impact on the considered response.

The linear terms of the factors showed an opposite effect on both considered responses. On the other hand, any change in one of the studied factors in order to improve one response had the opposite effect on the other one. For example, an increase in the peripheral speed improved the DA of the seeds, but reduced the WK percentage. Likewise, an increase in moisture content of the seeds in order to achieve a higher WK production reduced the ability of the seeds to dehull (i.e., the DA of the seeds). This situation makes it difficult to optimize the dehulling system.

The regression models developed were used for each response in order to determine the specified optimum conditions. These regression models are valid only in the selected experimental domain, which was determined taking into account some economic and operational considerations of the industry, and quality characteristics of the seeds.

In order to visualize the effect of the independent variables on both responses ( $D A$ and $W K$ ), response surface plots were generated from the developed models. Figures 2 and 3 show the effect of both the moisture content of the seeds and the peripheral speed on DA and WK, respectively, for Mycogen 9338 confectionary sunflower seeds.

$D A$ of the seeds increased with the increase in PS for all the seed moisture content. The increase in Dehulling efficiency with increasing PS at any moisture content of the seeds was also observed for an oilseed sunflower variety (Morden) [10], safflower seeds [9] and oat genotypes [15]. The highest DA at higher PS may be attributed to a higher discharge velocity of the seed and inducing a larger impact against the casing of the dehuller.

Decreasing MC caused an increase in DA on confectionary sunflower seeds in agreement with the reported for other sunflower varieties [8] and safflower seeds [9].

On the other hand, PS had negative linear and quadratic effects on WK, while MC had a positive linear effect on WK. Therefore, WK was found to increase as both the MC increased and PS decreased. The higher WK at low MC may be attributed to the kernel becoming brittle and thus, it broken easier. 


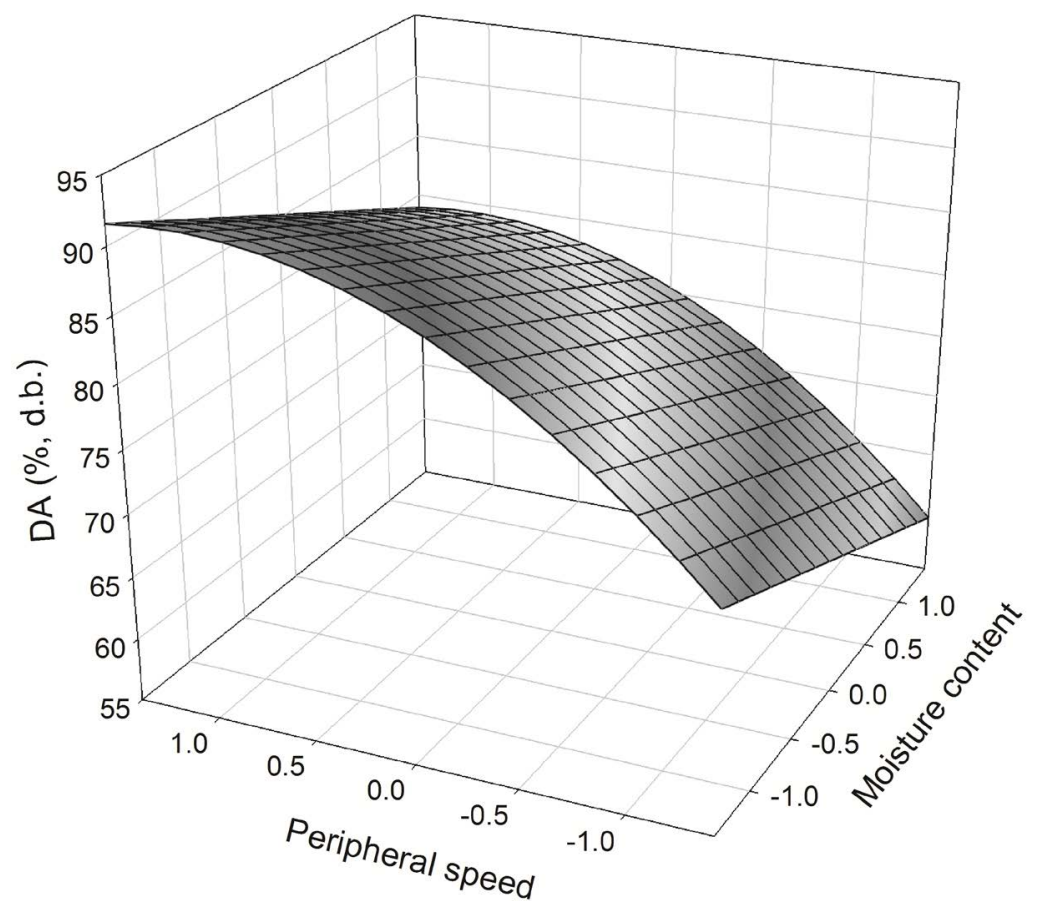

Figure 2. Effect of moisture content of the grains and peripheral speed on dehulling ability (DA).

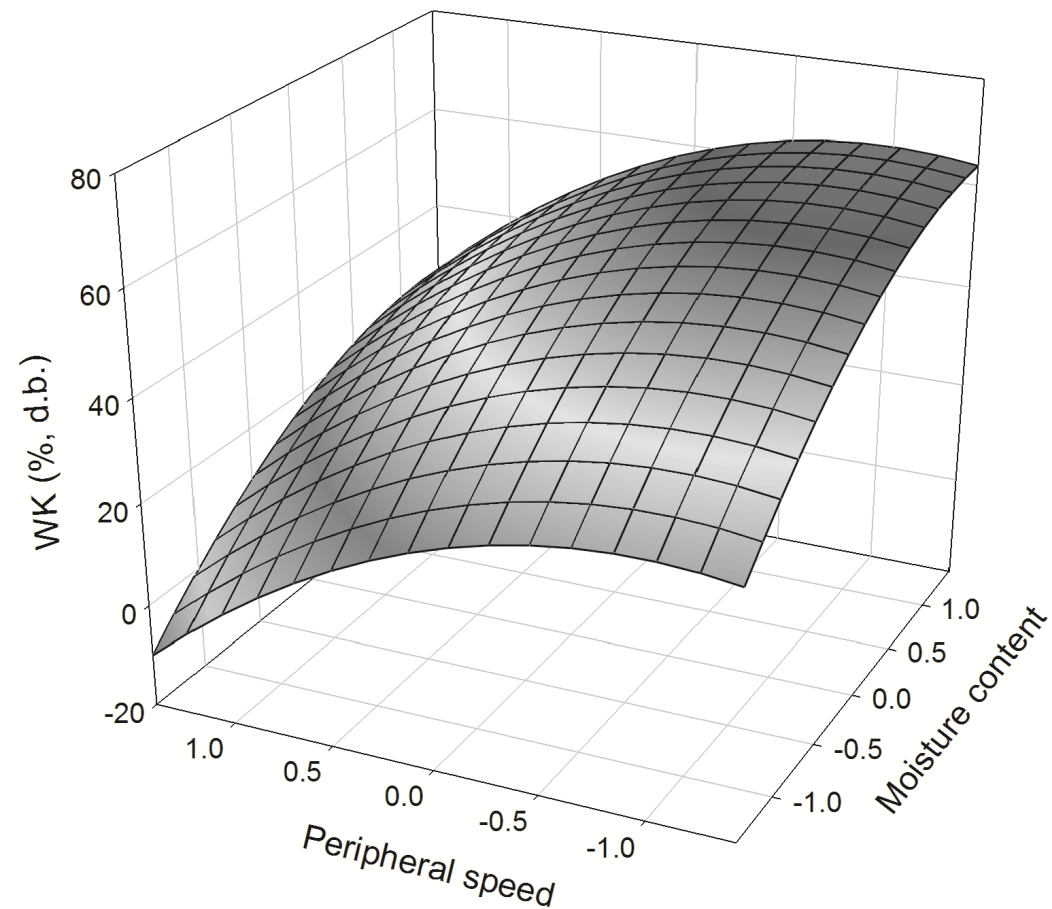

Figure 3. Effect of moisture content of the grains and peripheral speed on percentage of whole kernel (WK).

\subsection{Optimization}

Optimization of the processing operations should be performed in order to obtain the best conditions for the de- 
hulling of seeds, resulting in a superior quality product as well as maximizing throughput capacity.

By applying the desirability function method, the optimal operating conditions, obtained with a desirability value of 0.9460 , were $12.3 \%$ d.b. for the moisture content of the seeds and a peripheral speed of $32.5 \mathrm{~m} / \mathrm{s}$ for the dehuller equipment. This factor-level combination would achieve the maximum response values $(72.6 \%$ and 63.2\% for $D A$ and $W K$, respectively) for Mycogen 9338 confectionary sunflower seeds.

\subsection{Confirmatory Studies}

Verification experiment was performed at the optimum conditions obtained from the above study. The experimental $D A$ and $W K$ at the optimal operating conditions were $74.15 \% \pm 4.31 \%$ and $61.21 \% \pm 1.90 \%$, respectively. It was observed that experimental optimal values were reasonably close to the predicted values confirming the validity and adequacy of the predicted models.

\section{Conclusions}

The models developed in the present work could be used to predict the variation of $D A$, as a measure of the easiness of the seeds to dehull, and $W K$, in terms of both the moisture content of the seeds and impact speed for Mycogen 9338 confectionary sunflower seeds. The response surface methodology and desirability function method were used to determine the optimal processing conditions within the experimental region. The results of the optimization technique showed that the optimal conditions that maximize $D A$ and $W K$ could be obtained if the system is operated at a peripheral speed of $32.5 \mathrm{~m} / \mathrm{s}$ with a seed moisture content close to $12.3 \%$ d.b. Under these conditions, the values of $D A$ and $W K$ would be $72.6 \%$ and $63 \%$, respectively.

Taking into account that confectionary sunflower seeds should not be stored with a moisture content above $10 \%$ - $11 \%$, the optimum moisture value obtained from the experimental data (12.3\%, d.b.) determined the need to moisturize the seeds prior to the dehulling process. Hence, further studies of technical and economic feasibility may be necessary.

The results obtained offer criteria for the dehulling process of confectionary sunflower seeds, especially for the oil industry. This would help to optimize the process, and obtain products of better quality and higher profits.

\section{Acknowledgements}

The authors acknowledge the financial support from Universidad Nacional del Centro de la Provincia de Buenos Aires, Argentina.

\section{References}

[1] Tranchino, L., Melle F. and Sodoni, G. (1984) Almost Complete Dehulling of High Oil Sunflower Seed. Journal of the American Oil Chemists' Society, 6, 1261-1265. http://dx.doi.org/10.1007/BF02636267

[2] Subramanian, R., Shamanthaka Sastry, M.C. and Venkateshmurthy, K. (1990) Impact Dehulling of Sunflower Seeds: Effect of Operating Conditions and Seed Characteristics. Journal of Food Engineering, 12, 83-94. http://dx.doi.org/10.1016/0260-8774(90)90021-Y

[3] Merrien, A., Domínguez, J., Vannozzi, G.P., Baldini, M., Champolivier, L. and Carré, P. (1992) Factors Affecting the Dehulling Ability in Sunflower. Proceedings of the 13th International Sunflower Conference, Pisa, 260-267.

[4] Dedio, W. and Dorrel, D.G. (1989) Factors Affecting the Hullability and Physical Characteristics of Sunflower Achenes. Canadian Institute of Food Science and Technology Journal, 22, 143-146.

[5] Denis, L., Cohelo, V. and Vear F. (1994) Pericarp Structure and Hullability in Sunflower Inbred Lines and Hybrids. Agron, 14, 453-461. http://dx.doi.org/10.1051/agro:19940704

[6] Beauguillaume, A. and Cadeac, F. (1992) Elements of Explication of the Variability of the Hulling Ability in Sunflower. Proceedings of the 13th International Sunflower Conference, Pisa, 993-999.

[7] Riccobene, I.C., Fernández, M.B. and Nolasco, S.M. (2001) Efecto de la Humedad de la Semilla en la Aptitud al Descascarado de Frutos de Girasol (Helianthus annuus L.). Computer and Information Technology, 12, 3-8.

[8] de Figueiredo, A.K., Baümler, E., Riccobene, I.C. and Nolasco, S.M. (2011) Moisture-Dependent Engineering Properties of Sunflower Grains with Different Structural Characteristics. Journal of Food Engineering, 102, 58-65. http://dx.doi.org/10.1016/j.jfoodeng.2010.08.003 
[9] de Figueiredo, A.K., Rodríguez, L.M., Lindström, L.I., Riccobene, I.C. and Nolasco, S.M. (2013) Performance Analysis of a Dehulling System for Safflower Grains. Industrial Crops and Products, 43, 311-317. http://dx.doi.org/10.1016/j.indcrop.2012.07.026

[10] Gupta, R.K. and Das, S.K. (1999) Performance of Centrifugal Dehulling System for Sunflower Seeds. Journal of Food Engineering, 42, 191-198. http://dx.doi.org/10.1016/S0260-8774(99)00119-3

[11] (1999) Standard Engineering Practices Data, ASAE Standards, 46th Edition. American 440 Society of Agricultural Engineers, St. Joseph.

[12] del Castillo, E., Montgomery, D.C. and McCarville, D.R. (1996) Modified Desirability Functions for Multiple Response Optimization. Journal of Quality Technology, 28, 337-345.

[13] Eren, I. and Kaymak-Ertekin, F. (2007) Optimization of Osmotic Dehydration of Potato Using Response Surface Methodology. Journal of Food Engineering, 79, 344-352. http://dx.doi.org/10.1016/j.jfoodeng.2006.01.069

[14] Corzo, O., Bracho, N., Vásquez, A. and Pereira, A. (2008) Optimization of a Thin Layer Drying Process for Coroba Slices. Journal of Food Engineering, 85, 372-380. http://dx.doi.org/10.1016/j.jfoodeng.2007.07.024

[15] Doehlert, D.C., Wiesenborn, D.P, McMullen, M.S., Ohm, J.B. and Riveland, N.R. (2009) Effects of Impact Dehuller Rotor Speed on Dehulling Characteristics of Diverse Oat Genotypes Grown in Different Environments. Cereal Chemistry, 86, 653-660. http://dx.doi.org/10.1094/CCHEM-86-6-0653

\section{Abbreviations}

CCRD: Central composite rotatable design

DA: Dehulling ability

D: Desirability function

Db: Dry basis

MC: Moisture content of the seeds

PS: Peripheral speed

RSM: Response surface methodology

WK: Whole kernel 